\title{
Prevention of Adjacent Segmental Disease after Fusion in Degenerative Spinal Disorder: Correlation between Segmental Lumbar Lordosis Ratio and Pelvic Incidence-Lumbar Lordosis Mismatch for a Minimum 5-Year Follow-up
}

\author{
Whoan Jeang Kim, Chang Hyun Ma, Sang Ha Kim, Yeon Seung Min, Jae Won Lee, \\ Shann Haw Chang, Kyung Hoon Park, Kun Young Park, Dae Gun Song, Won Sik Choy \\ Department of Orthopedic Surgery, Eulji University Hospital, Daejeon, Korea
}

\section{Study Design: Retrospective study.}

Purpose: Associations among risk factors related to adjacent segmental disease (ASD) remain unclear. We evaluated the risk factors and segmental lordosis ratio to prevent ASD developing after lumbar spinal fusion.

Overview of Literature: Risk factors related to ASD development are age, sex, obesity, pre-existing degeneration, number of fusion segments, and decreased postoperative lumbar lordosis (LL). However, the associations among these factors are still unclear and should be clearly identified.

Methods: We retrospectively reviewed data on 274 patients who underwent lumbar spinal fusion of three segments or below for lumbar degenerative disease from January 2010 to December 2012, with over 5 years of follow-up. Patients with preoperative sagittal vertical axis (SVA) $>5 \mathrm{~cm}$ were excluded due to sagittal imbalance. A total of 37 patients with ASD and 40 control patients (CTRL) were randomly selected in a similar distribution of matching variables: age, sex, and preoperative degenerative changes. Sex, age, number of fusion segments, radiologic measurements, L4-5-S1/L1-S1 LL ratio, and spinopelvic parameters (pelvic incidence [PI], pelvic tilt [PT], sacral slope [SS], and SVA) were analyzed. Logistic regression was used to analyze the correlation between PI-LL mismatch and L4-5-S1 segmental lordosis rate.

Results: No significant difference was found between ASDs and CTRL groups regarding age, sex, number of fusion segments, fusion method, and preoperative and postoperative spinopelvic parameters (PI, SS, PT, and LL). However, regarding the L4-5-S1/L1-S1 lordosis ratio, $50 \%(p=0.045), 60 \%(p=0.031), 70 \%(p=0.042), 80 \%(p=0.023)$, and $90 \%(p=0.023)$ were statistically significant; $<20 \%$ $(p=0.478), 30 \%(p=0.223)$, and $40 \%$ ( $p=0.089)$ were not statistically significant. In the postoperative PI-LL $<10$ group, ASD occurred less frequently than in the PI-LL $>10$ group, and the difference was statistically significant $(p=0.048)$.

Conclusions: Patients with a postoperative L4-5-S1/L1-S1 lordosis ratio $>50 \%$ had less occurrence of ASD. Correcting LL according to $\mathrm{Pl}$ and physiologic segmental lordosis ratio is important in preventing ASD.

Keywords: Adjacent segmental disease; Lumbar lordosis; Lumbar degenerative disease; Segmental lordosis ratio; Spinopelvic parameters 


\section{Introduction}

Spinal fusion has become a common treatment for numerous pathologic conditions of the human spine [1-4]. However, spinal fusions may increase the stress on the nonoperated adjacent lumbar segments, causing a common complication of adjacent segmental degeneration in long-term follow-up [5-7].

Cheh et al. [8] found that $43 \%$ of patients showed radiographic adjacent segmental degeneration and $24 \%$ had symptomatic adjacent segmental disease (ASD), whereas $6.3 \%$ had clinical signs without radiographic evidence. The reported ASD rates in the literature range from $2.6 \%$ to $30.3 \%[2,9]$. We used the term ASD for symptomatic adjacent segmental degeneration. The development of ASD is problematic because it can necessitate further surgical intervention and adversely affect functional outcomes [9]. For this reason, there has recently been a growing interest in the risk factors to prevent and treat degenerative changes in adjacent segments after spinal fusion. Known risk factors related to the development of ASD were age, sex, obesity, pre-existing degeneration, number of fusion segments, and decreased postoperative lumbar lordosis (LL) $[2,4,10,11]$. However, the associations among these factors remain unclear and should be clearly identified.

The importance of spinopelvic balance and its implications on the clinical treatment of patients with lower back pain have been shown in recent studies [12-15]. However, little is known about how spinopelvic alignment affects adjacent segmental stress or how this may contribute to lumbar disk degeneration. Since the relationship between pelvic incidence (PI) as a morphologic parameter and LL appears important for the sagittal profile of the spine, it may also account for different loading patterns in the lumbar spine, which may be relevant for the development of adjacent segmental degeneration and disease. Rothenfluh et al. [16] demonstrated that patients with $\Delta \mathrm{PI}-\mathrm{LL}>10$ have a 10 times higher risk for ASD development, while a PI-LL mismatch of $\geq 10$ indicated that patients are likely to have either imbalance or compensating.

Furthermore, in terms of regional balance, Bernhardt and Bridwell [17] showed that $>60 \%$ of LL is created by the disks at L4-5 and L5-S1 in the normal spine, which contribute $-20^{\circ}$ and $-28^{\circ}$ to the regional lordotic measurement. The normal range of the total lumbar lordotic angle according to Jackson and McManus [18] is $33^{\circ}-88^{\circ}$ (L1-S1) and $33^{\circ}-79^{\circ}$ (L1-S1) according to Peterson et al.
[19] Kim et al. [20] analyzed the normal mean value of LL in the Asian spine, which was $47^{\circ}$, ranging from $23^{\circ}-65^{\circ}$. However, LL has a large standard deviation and different normal values for each individual; hence, it is inappropriate to compare. We used ratio to discuss regional lordosis. We focused on correcting L4-5-S1 segmental lordosis based on the above finding to significantly reduce the incidence of ASD.

To the best of our knowledge, no study has demonstrated the correlation between PI-LL mismatch and segmental lordosis ratio (L4-5-S1/L1-S1 lordosis ratio) for the prevention of ASD.

\section{Materials and Methods}

We hypothesized that PI-LL $<10$ and correction of L4-5S1 segmental lordosis ratio to physiologic lordosis may prevent the incidence of ASD. This study was retrospective in nature and we obtained final approval of exemption from Eulji University Hospital institutional review board. and the written informed consets were obtained.

A total of 274 adults with lumbar spinal degeneration surgically treated at a single institution between January 2010 and December 2012 were studied. All patients were followed for a minimum of 5 years. Inclusion criteria were patients who underwent primary lumbar fusion of three segments or lower between L2 and S1 for lumbar degenerative disease and underwent revision surgery for symptomatic ASD during follow-up. All study patients underwent standing whole-spine lateral X-ray with inclusion of the femoral heads. Indications for surgery were degenerative lumbar disorders with leg pain and claudication. Our exclusion criteria were patients who underwent previous surgery and had been followed for $<5$ years postoperatively patients with a preoperative sagittal vertical axis (SVA) $>5 \mathrm{~cm}$ were excluded because of sagittal imbalance. In addition, we excluded patients with degenerative lumbar scoliosis more severe than $20^{\circ}$.

Sagittal imbalance was measured by the standing wholespine lateral X-ray taken according to the method recommended by the preoperative spinal deformity study group. Magnetic resonance imaging (MRI) was performed in patients with back pain and neurogenic claudication and showed improvement in symptoms for a certain period of time after initial surgery to exclude cases of degenerative changes in adjacent segments without clinical symptoms. ASD was diagnosed when spinal canal stenosis and disk 
herniation were observed at the adjacent segment on MRI. The patient underwent revision surgery for at least 3 months in which symptoms persisted.

Of 274 patients, 40 control patients (CTRL) were randomly selected from the patient pool of lumbar spinal fusion procedures that met the above inclusion criteria, except for revision surgery, to be similar in the distribution of age, sex, and preoperative degenerative changes to those of the ASDs group with the same exclusion criteria. Patients were included in the CTRL group only if they had a minimum follow-up of 5 years, no signs of symptomatic ASD at last follow-up, similar distribution of levels and number of segments fused, and a comparable degree of disk degeneration in the prospective adjacent segment preoperatively as assessed on MRI.

In total, 77 patients (37 ASDs, 40 CTRL) were included who demonstrated adequate follow-up and radiographs with preoperative MRI of the lumbar spine. All patients who underwent posterolateral instrumented fusion with pedicle screws showed union during follow-up. Details on the patients' demographic data are given in Table 1. The Pfirrmann grade was measured to prove that there was no difference in the degree of preoperative disk degeneration between ASDs and CTRLs and the results demonstrated there was no significant difference between the two groups (Table 2). Similar groups in the distribution of their matching variables were selected before measurements were performed on radiographs to minimize the selection bias.

Sex, age, number of fusion segments, and radiological

Table 1. Demographic data of patients $(\mathrm{N}=77)$

\begin{tabular}{lcc} 
Characteristic & ASDs $(\mathrm{n}=37)$ & $\begin{array}{c}\text { Control patients } \\
(\mathrm{n}=40)\end{array}$ \\
\hline Follow-up (mo) & $72.2 \pm 9.7(61-96)$ & $76.8 \pm 8.8(61-94)$ \\
\hline Age $(\mathrm{yr})$ & $69.6 \pm 7.5(54-83)$ & $65.6 \pm 11.1(55-81)$ \\
\hline Sex (male:female) & $14: 23$ & $16: 24$ \\
\hline Body mass index $\left(\mathrm{kg} / \mathrm{m}^{2}\right)$ & $28.9 \pm 3.8$ & $27.8 \pm 4.0$ \\
Fusion segment & & \\
1 & 20 & 23 \\
\hline 2 & 13 & 14 \\
3 & 4 & 3 \\
\hline Revision for ASD (mo) & $44(6-85)$ & - \\
\hline
\end{tabular}

Values are presented as mean \pm standard deviation (range), number, mean \pm standard deviation, or number (range).

ASD, adjacent segmental disease. measurements, including segmental lordosis ratio and pelvic parameters, were analyzed. The spinopelvic alignment was characterized by LL, PI, sacral slope (SS), and pelvic tilt (PT) values. The differences between PI and LL were computed, and for the grouping of patients, a PI-LL threshold of 10 was adopted based on previous studies.

LL was measured from L1-S1 and L4-S1 for the assessment of L4-5-S1/L1-S1 segmental lordosis ratio (Fig. 1). In addition, according to the number of fusion segments after the first operation, when fusion was performed from the L3 level, the lordotic angle was measured between L3 upper end plate and S1; when the fusion was performed from the L4 level, the lordotic angle was measured between L4 upper end plate and S1. The results were compared by $>30 \%, 40 \%, 50 \%, 60 \%$, and $70 \%$ (Table 3 ).

Table 2. Preoperative Pfirrmann grade of ASDs and CTRL group

\begin{tabular}{lcc} 
Pfirmann grade & ASDs $(n=37)$ & CTRL $(n=40)$ \\
1 & 5 & 4 \\
2 & 13 & 14 \\
3 & 11 & 12 \\
4 & 4 & 5 \\
5 & 4 & 5 \\
\hline
\end{tabular}

ASD, adjacent segmental disease; CTRL, control patients.

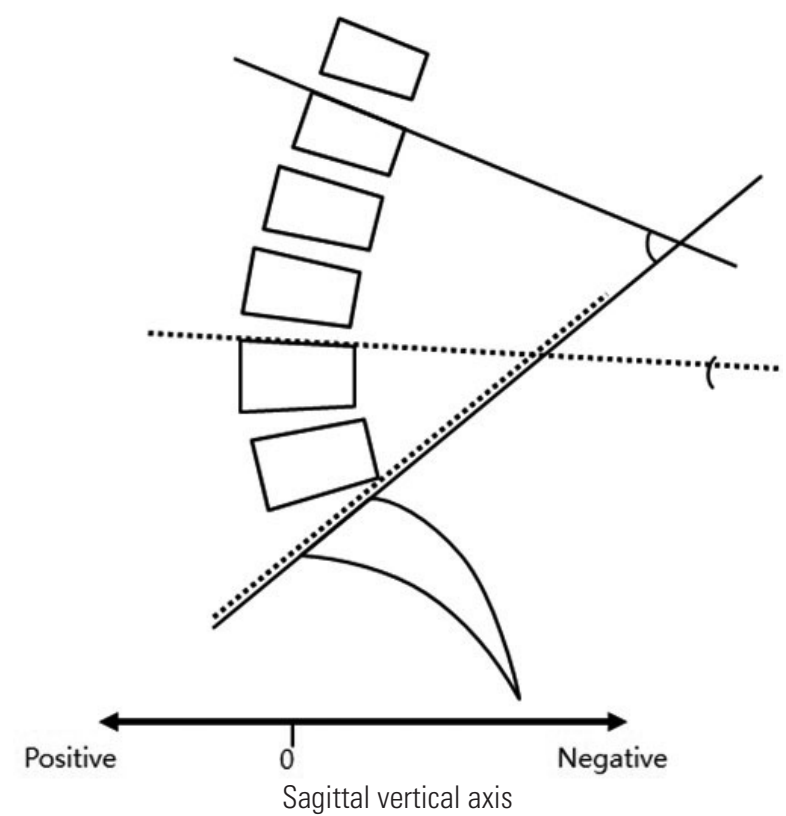

Fig. 1. Segmental lordosis ratio was performed as shown. The solid line indicates L1-S1 lumbar lordosis, the dotted line indicates L4-S1 lumbar lordosis 
Table 3. Correlation of postoperative pelvic parameter and segmental lordotic angle ratio ( $N=77)$

\begin{tabular}{|c|c|c|c|c|}
\hline \multirow{2}{*}{ L4-5-S1/L1-S1 (\%) } & \multicolumn{2}{|c|}{ Adjacent segmental disease $(n=37)$} & \multicolumn{2}{|c|}{ Control patients ( $n=40$ ) } \\
\hline & $\mathrm{Pl}-\mathrm{LL}<10(\mathrm{n}=12)$ & $\mathrm{PI}-\mathrm{LL}>10(\mathrm{n}=25)$ & $\mathrm{Pl}-\mathrm{LL}<10(\mathrm{n}=24)$ & $\mathrm{PI}-\mathrm{LL}>10(\mathrm{n}=16)$ \\
\hline$<20$ & 1 & 2 & 1 & 1 \\
\hline $20-30$ & 2 & 3 & 1 & 2 \\
\hline 30-40 & 3 & 5 & 2 & 1 \\
\hline $40-50$ & 3 & 8 & 5 & 2 \\
\hline $50-60$ & 1 & 3 & 10 & 4 \\
\hline $60-70$ & 1 & 2 & 3 & 3 \\
\hline $70-80$ & 1 & 1 & 1 & 2 \\
\hline$>80$ & 1 & 0 & 1 & 1 \\
\hline
\end{tabular}

$\mathrm{PI}$, pelvic incidence; LL, lumbar lordosis.

Table 4. Levels, number of segments fused, and fusion methods ( $\mathrm{N}=77$ )

\begin{tabular}{|c|c|c|c|}
\hline Variable & Adjacent segmental disease $(n=37)$ & Control patients $(\mathrm{n}=40)$ & $p$-value \\
\hline \multicolumn{4}{|l|}{ Levels (no. of segments fused) } \\
\hline L2-5 (3) & 2 & 2 & \\
\hline $\mathrm{L} 3-4(1)$ & 3 & 4 & \\
\hline $\mathrm{L} 3-5(2)$ & 5 & 4 & \\
\hline$\llcorner 4-5(1)$ & 17 & 22 & \\
\hline L3-S1 (3) & 2 & 2 & \\
\hline L4-S1 (2) & 5 & 4 & \\
\hline L5-S1 (1) & 3 & 2 & \\
\hline \multicolumn{4}{|l|}{ Fusion methods } \\
\hline Posterior lumbar fusion only & 23 & 28 & 0.590 \\
\hline Lumbar interbody fusion (1 level) & 12 & 10 & 0.684 \\
\hline Posterior lumbar interbody fusion (2 level) & 2 & 2 & 0.854 \\
\hline
\end{tabular}

Radiographic measurements were performed using m-view (Infinity Healthcare Co., Ocean City, NJ, USA) on the Hospital's Picture Archiving and Communication System. Measurements were obtained twice for each author and the mean values were calculated. Reliability was classified as small (0-0.24), low (0.25-0.49), medium (0.50-0.69), excellent (0.70-0.89), and best (0.90-1.0) depending on the coincidence coefficient in the group. The interobserver reliability of this study was estimated to be 0.93 .

Statistical analysis was performed using PASW SPSS ver. 18.0 (SPSS Inc., Chicago, IL, USA) for the two groups. The Mann-Whitney $U$-test and the two-sample Kolmogorov-Smirnov test were used to confirm the statistical significance of the measurements in both groups. We used logistic regression analysis to analyze the correlation between PI-LL mismatch and L4-5-S1 segmental lordosis rate. The significance level was $p<0.05$

\section{Results}

No statistically significant difference was found between the ASD and CTRL groups on age, sex, body mass index, and number of fusion segments (Tables 1,2). Among the fusion methods, there were 23 cases of posterolateral fusion, 12 one-level posterior lumbar interbody fusion (PLIF), and two two-level PLIF in the ASD group, and 28, 10 , and two, respectively, in the CTRL group. Moreover, no statistically significant difference was found between the two groups (Table 4). 
Table 5. Radiographic measurements

\begin{tabular}{|c|c|c|c|c|c|c|}
\hline \multirow{2}{*}{ Variable } & \multicolumn{3}{|c|}{ Adjacent segmental disease $(n=37)$} & \multicolumn{3}{|c|}{ Control patients ( $\mathrm{n}=40)$} \\
\hline & Preop & Postop & $p$-value & Preop & Postop & $p$-value \\
\hline L1-S1 lumbar lordosis ( $\left(^{\circ}\right)$ & $40.6 \pm 9.5$ & $40.7 \pm 11.8$ & 0.131 & $41.8 \pm 10.1$ & $45.2 \pm 10.8$ & 0.072 \\
\hline L4-5-S1 segmental lordosis $\left({ }^{\circ}\right)$ & $21.3 \pm 9.1$ & $19.2 \pm 9.4$ & 0.114 & $22.1 \pm 8.4$ & $24.8 \pm 7.7$ & 0.241 \\
\hline L4-5-S1/L1-S1 (\%) & $51.7 \pm 1.9$ & $47.4 \pm 1.7$ & 0.135 & $53.5 \pm 1.3$ & $54.8 \pm 1.4$ & 0.422 \\
\hline $\mathrm{PI}-\mathrm{LL}\left({ }^{\circ}\right)$ & $15.1 \pm 12.3$ & $13.1 \pm 13.4$ & 0.279 & $12.8 \pm 9.3$ & $7.3 \pm 9.3$ & 0.035 \\
\hline Pelvic tilt $\left({ }^{\circ}\right)$ & $23.9 \pm 8.5$ & $22.3 \pm 8.7$ & 0.275 & $21.4 \pm 8.8$ & $21.3 \pm 7.7$ & 0.214 \\
\hline Sacral slope $\left({ }^{\circ}\right)$ & $31.1 \pm 6.6$ & $31.5 \pm 8.1$ & 0.301 & $30.5 \pm 7.1$ & $32.1 \pm 8.6$ & 0.355 \\
\hline Pelvic incidence $\left({ }^{\circ}\right)$ & $54.9 \pm 9.4$ & & & $52.3 \pm 11.3$ & & \\
\hline Sagittal vertical axis $(\mathrm{cm})$ & $2.4 \pm 1.3$ & $2.1 \pm 1.8$ & 0.873 & $2.5 \pm 1.1$ & $2.3 \pm 1.1$ & 0.741 \\
\hline
\end{tabular}

Values are presented as mean \pm standard deviation.

Preop, preoperative; Postop, postoperative.
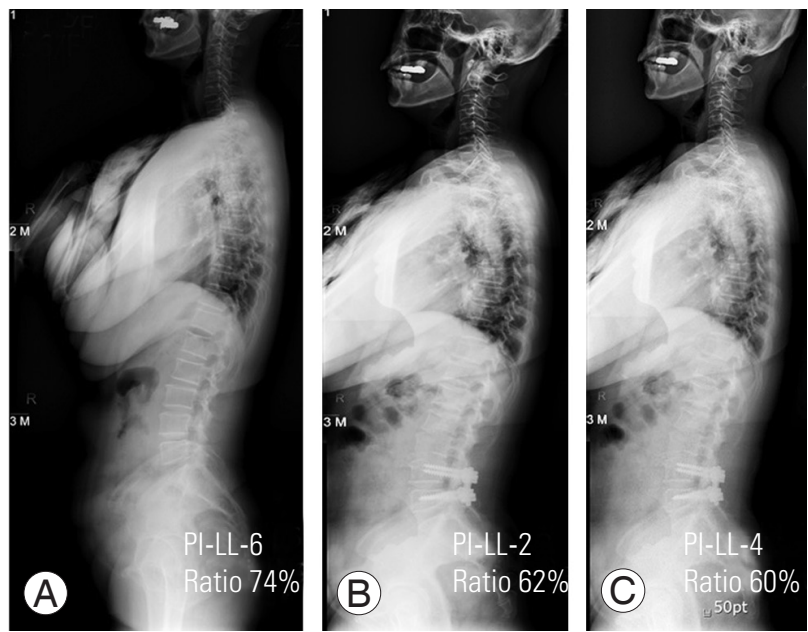

Fig. 2. (A) Preoperative radiograph; (B) 2 weeks postoperatively; and (C) final follow-up 4 years 8 months postoperatively. A 56-year-old woman with one segment fusion. Preoperative measurements were PI-LL -6, segmental lordotic ratio 74\%. Postoperative measurements were $\mathrm{PI}-\mathrm{LL}-2$, segmental lordotic ratio $62 \%$. Patient with $\mathrm{PI}-\mathrm{LL}<10$ and good segmental lordotic ratio showed a good prognosis without adjacent segmental disease. PI, pelvic incidence; LL, lumbar lordosis.

On our radiologic measurements, the comparison of pre- and postoperative values showed no significant differences in terms of spine parameter between the ASD and CTRL groups for L1-S1 LL and L4-5-S1 LL. No significant differences were found between the two groups regarding pelvic or postoperative parameters. However, PI-LL in the CTRL group showed a statistically significant difference between preoperative and postoperative values (Table 5).

We analyzed the correlation between L4-5-S1 seg-
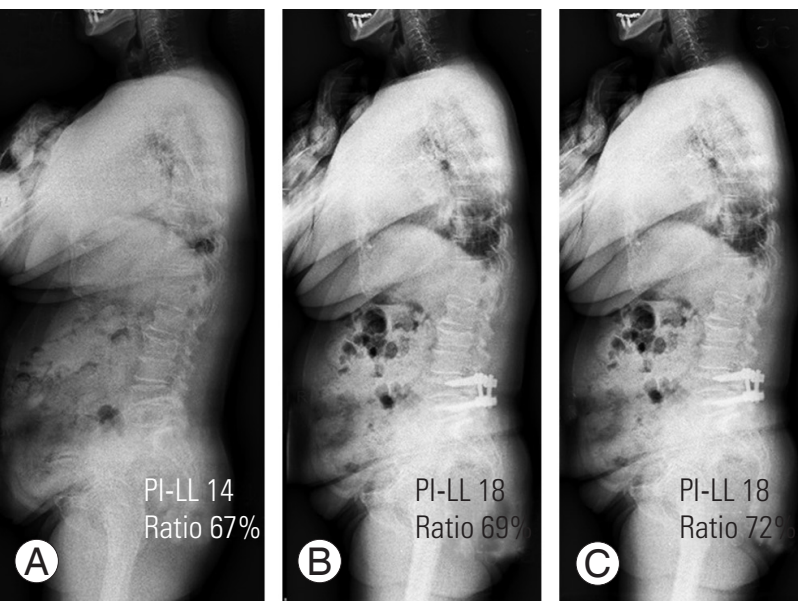

Fig. 3. (A) Preoperative radiograph; (B) 2 years 3 months postoperatively; and (C) postoperative radiograph of revision surgery. A 72-yearold woman with one segment fusion. Preoperative measurements were PI-LL 14, segmental lordotic ratio 67\%. Postoperative measurements were PI-LL 18, segmental lordotic ratio 69\%. Patient with Pl$L L>10$, but good segmental lordotic ratio showed good prognosis and underwent revision surgery. PI, pelvic incidence; LL, lumbar lordosis.

mental lordosis ratio and ASD. The results were $>30 \%$ $(p=0.223), 40 \%(p=0.089), 50 \%(p=0.045), 60 \%(p=0.031)$, $70 \%(p=0.042)$. When the ratio was $>50 \%(p=0.045)$, a statistical significance was observed and ASD occurred less frequently when the L4-5-S1 segmental lordosis ratio was $>50 \%$ (Table 3, Figs. $2-5$ ).

PI-LL was $<10$ in 12 and 24 patients and $>10$ in 25 and 16 in the ASD $(n=37)$ and CTRL $(n=40)$ groups, respectively. Of the 77 patients, the rate of ASD occurrence was $33.3 \%(12 / 36)$ in the postoperative PI-LL $<10$ group 

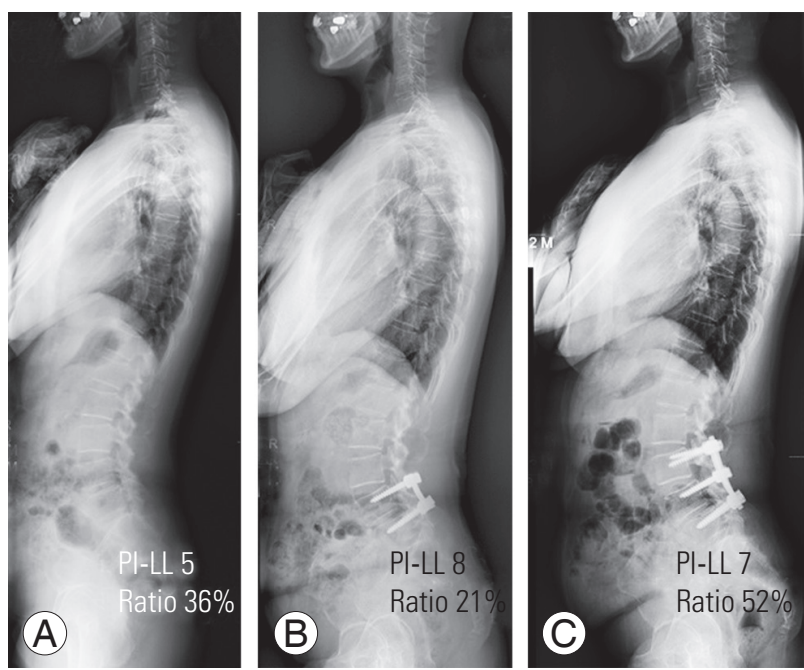

Fig. 4. (A) Preoperative radiograph. (B) 2 years 3 months postoperatively. (C) Postoperative radiograph of revision surgery. A 63-year-old woman with 1 segment fusion and posterior lumbar interbody fusion. Preoperative measurements were $\mathrm{PI}-\mathrm{LL}$ 5, segmental lordotic ratio $36 \%$. Postoperative measurements were PI-LL 8, segmental lordotic ratio $21 \%$. Patient with $\mathrm{PI}-\mathrm{LL}<10$, but poor segmental lordotic ratio showed poor prognosis and underwent revision surgery. PI, pelvic incidence; LL, lumbar lordosis.
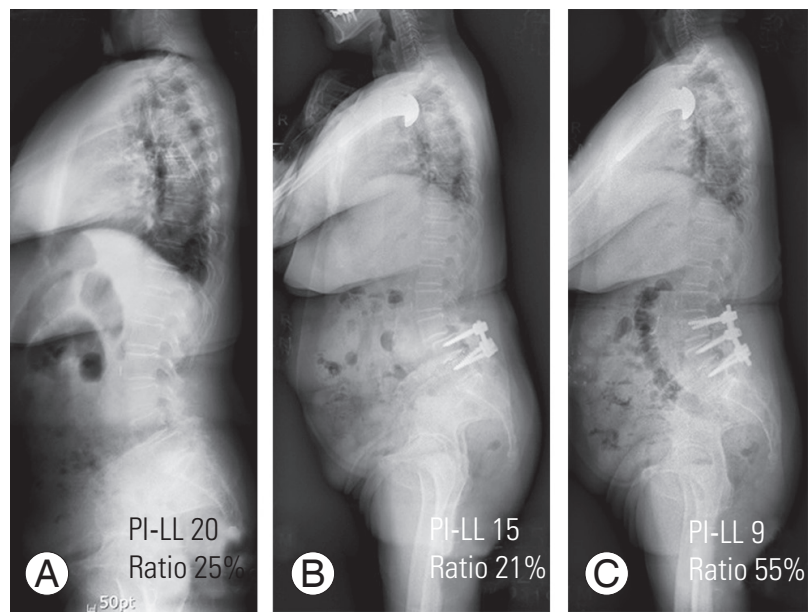

Fig. 5. (A) Preoperative radiograph; (B) 6 months postoperatively; and (C) postoperative radiograph of revision surgery. A 75-year-old woman with one segment fusion. Preoperative measurement were PI-LL 20, segmental lordotic ratio 25\%. Postoperative measurements were $\mathrm{Pl}-$ $\mathrm{LL} 15$, segmental lordotic ratio $21 \%$. Patient with $\mathrm{PI}-\mathrm{LL}>10$ and poor segmental lordotic ratio showed poor prognosis and underwent revision surgery. The patient had an ASD at 6 months postoperatively and underwent revision at the early stage. This case suggested that if the $\mathrm{PI}-\mathrm{LL}$ and the regional lordosis were not aligned well, the ASD could occur more rapidly. PI, pelvic incidence; LL, lumbar lordosis; ASD, adjacent segmental disease.

and $61 \%(25 / 41)$ in the PI-LL $>10$ group. In the postoperative PI-LL $<10$ group, ASD occurred less than in the $\mathrm{PI}-\mathrm{LL}>10$ group, and this was statistically significant $(p=0.048)$ (Table 3$)$.

In the PI-LL $<10$ group, if the segmental lordosis ratio was $<50 \%$, ASD occurred frequently (ASD group, $75 \%$ [9/12]; CTRL group, 37.5\% [9/24]). However, in the PILL $>10$ group, if the lumbar segmental lordosis ratio was $>50 \%$, ASD occurred less frequently (ASD group, $24 \%$ [6/25]; CTRL group, $62.5 \%$ [10/16]). We found that the incidence of ASD was significantly reduced in patients with a PI-LL $<10$ and a L4-5-S1/L1-S1 segmental lordosis ratio of $>50 \%$ (Table 3 ).

\section{Discussion}

Hilibrand and Robbins [5] described ASD as a radiologic change in the adjacent segment only and a clinical symptom accompanied by radiologic change. Recently, many studies were done on the risk factors related to ASD after fusion [1-4,10,11]. The development of ASD is problematic and adjacent segmental degeneration and disease have a significant clinical impact; hence, numerous studies have aimed at identifying the risk factors [9].

The risk factors for ASD development include patient factors, such as age, sex, and bone mineral density; preoperative factors, such as instability and intervertebral disc herniation; and postoperative factors, such as sagittal alignment, number of fusion segments, and fusion method $[1-4,10,11]$. However, the risk factors with relatively clear implications on subsequent surgical management have not been identified.

Many researchers thought that there would be more changes in the adjacent segments with increasing age. Lee et al. [2] reported that the incidence of ASD was higher in patients $>60$ years old, and Aota et al. [21] found that the incidence of ASD was higher in patients $>55$ years old. However, controversies exist regarding the relationship between the occurrence of segmental diseases and age [4]. In our study, no statistically significant difference was noted ( $p=0.424$ ) between the mean age of patients with (72.2 \pm 9.7 years) and without (76.8 \pm 8.8 years) ASD. Although older ages are likely to influence the development of ASD, considering that both groups are $>60$ years old, it would be unreasonable to evaluate that older age affects ASD in this study.

Kim et al. [22] reported no significant differences between the posterior interbody fusion and posterolateral fusion in the incidence of ASD. However, Lee et al. [3] and Brodsky et al. [23] compared posterior interbody fusion 
and posterolateral fusion, and the incidence of ASD was lower in posterolateral fusion. In our study, no significant difference was noted between posterior interbody fusion and posterolateral fusion in the incidence of ASD (Table 4).

Furthermore, no statistically significant difference was found between the ASD (1.48 \pm 0.34$)$ and CTRL groups $(1.40 \pm 0.27)$ in terms of fusion segment number $(p=0.486)$. Moreover, no correlation between fusion segment number and occurrence of ASD was found in patients with threesegment fusion or less.

In addition, there is a correlation that the incidence of ASDs is increased because stress is highly concentrated on the adjacent segments as multiple segments are fused. Kim et al. [1] and Rohlmann et al. [24] reported that the cause of increased compensatory motion of adjacent segments in the long segmental fusion was more influenced by sagittal or coronal imbalance than by physical factors. As the importance of LL correction is emphasized when long segment fusion is needed, performing anterior fusion together is becoming increasingly common.

PI is a unique radiologic index and predictor of the amount of LL required to assume a balanced sagittal posture. It is a very important factor in evaluating sagittal balance [18]. Recently, Schwab et al. [25] reported sagittal modifiers, including SVA, PT, and PI-LL, which are indicators of health-related quality of life. The International Spine Study Group has recently reported the relevance of PI-LL mismatch in adult deformity. A significant difference was found between these indicators and thus their correction is an important factor in surgical treatment. However, studies on altered kinematics and biomechanics after lumbar spinal fusion did not focus on spinopelvic alignment or sagittal imbalance, but instead investigated hypermobility and increased loads in the adjacent segment [16]. The appropriate lumbar lordotic angle is known as $\mathrm{PI} \pm 9^{\circ}$. Rothenfluh et al. [16] demonstrated that patients with $\triangle \mathrm{PI}-\mathrm{LL}>10$ have a 10 times higher risk for ASD development and PI-LL mismatch of $\geq 10$ indicated that patients are either likely to have imbalance or compensating. In this study, the relationship between the degree of lumbar lordotic deviation and ASD occurrence was evaluated using PI, and when PI-LL was $<10$, it was statistically significant $(p=0.048)$. Other spinopelvic parameters, including SS, PT, and SVA, did not show a statistically significant difference because we excluded patients with sagittal imbalance.
In terms of regional lordosis, Bernhardt and Bridwell [17] reported that LL $>60 \%$ is created by L4 -5 and L5-S1 disks. In the present study, when L4-5-S1/L1-S1 segmental lordosis ratio was approximately $50 \%$, ASD occurred less frequently. We believe that LL has a large standard deviation and different normal values for each individual; hence, it is inappropriate to compare and so we used ratio to discuss regional lordosis. Considering functional neutrality, a statistically significant result could be obtained in a wider range than the idea that occurrence of ASD would be less frequent if the ratio was $60 \%$. When the ratio was $50 \%(p=0.045)$, a statistical significance could be observed. We believe that is because our study subjects are an elderly population with normal progressive spinal degeneration; thus, $50 \%$ appears to be acceptable.

Sagittal alignment is important to prevent the development of ASD, and sagittal plane balance after lumbar fusion is an important factor that affects the motion of adjacent segments and is directly related to LL. In this study, when PI-LL was satisfactorily corrected to $<10$, but the lumbar segmental lordosis ratio was not, ASD frequently occurred in the corrected group (ASD group, 75\% [9/12]; CTRL group, 37.5\% [9/24]). We suggest that for reducing the incidence of ASD, lumbar segmental lordosis ratio should be evaluated as well as the appropriate LL angle according to each individual's PI.

The major limitation of this study was the lack of representation of the population as a whole, due to the small sample size and the fact that the patients were followed for $>5$ years, which is a long period of time.

L4 was not the only transition point in the lumbar spine [26]. That means that lower lumbar curvature may share less proportion in the whole lumbar spine in some cases. In surgical methods, we used only posterior lumbar fusion and PLIF to restore LL. Further studies were needed on LL restoration through surgical procedures, such as anterior lumbar, direct lateral, and transforaminal lumbar interbody fusion. In addition, it was difficult to determine the factors affecting the resultant value in various aspects. Moreover, evaluations of actual function, satisfaction, and other complications were insufficient. However, given that the evaluation was made only by radiologic measurement, relatively objective data could be obtained.

\section{Conclusions}

After a fusion operation for a degenerative spine, patients 
with a postoperative L4-5-S1/L1-S1 segmental lordosis ratio of $>50 \%$ experienced less frequent occurrences of ASD. Correcting the LL according to PI and physiologic lordosis ratio is important in preventing ASD.

\section{Conflict of Interest}

No potential conflict of interest relevant to this article was reported.

\section{References}

1. Kim WJ, Kang JW, Kam BS, et al. Analysis of risk factors and surgical results of lumbar adjacent segment disease. J Korean Soc Spine Surg 2010;17:74-81.

2. Lee CS, Hwang CJ, Lee SW, et al. Risk factors for adjacent segment disease after lumbar fusion. Eur Spine J 2009;18:1637-43.

3. Lee JC, Kim Y, Soh JW, Shin BJ. Risk factors of adjacent segment disease requiring surgery after lumbar spinal fusion: comparison of posterior lumbar interbody fusion and posterolateral fusion. Spine (Phila Pa 1976) 2014;39:E339-45.

4. Soh J, Lee JC, Shin BJ. Analysis of risk factors for adjacent segment degeneration occurring more than 5 years after fusion with pedicle screw fixation for degenerative lumbar spine. Asian Spine J 2013;7:27381.

5. Hilibrand AS, Robbins M. Adjacent segment degeneration and adjacent segment disease: the consequences of spinal fusion? Spine J 2004;4(6 Suppl):190S-194S.

6. Levin DA, Hale JJ, Bendo JA. Adjacent segment degeneration following spinal fusion for degenerative disc disease. Bull NYU Hosp Jt Dis 2007;65:29-36.

7. Xia XP, Chen HL, Cheng HB. Prevalence of adjacent segment degeneration after spine surgery: a systematic review and meta-analysis. Spine (Phila Pa 1976) 2013;38:597-608.

8. Cheh G, Bridwell KH, Lenke LG, et al. Adjacent segment disease following lumbar/thoracolumbar fusion with pedicle screw instrumentation: a minimum 5-year follow-up. Spine (Phila Pa 1976) 2007;32:22537.

9. Park P, Garton HJ, Gala VC, Hoff JT, McGillicuddy JE. Adjacent segment disease after lumbar or lumbosacral fusion: review of the literature. Spine (Phila Pa
1976) 2004;29:1938-44.

10. Hikata T, Kamata M, Furukawa M. Risk factors for adjacent segment disease after posterior lumbar interbody fusion and efficacy of simultaneous decompression surgery for symptomatic adjacent segment disease. J Spinal Disord Tech 2014;27:70-5.

11. Masevnin S, Ptashnikov D, Michaylov D, Meng H, Smekalenkov O, Zaborovskii N. Risk factors for adjacent segment disease development after lumbar fusion. Asian Spine J 2015;9:239-44.

12. Gottfried ON, Daubs MD, Patel AA, Dailey AT, Brodke DS. Spinopelvic parameters in postfusion flatback deformity patients. Spine J 2009;9:639-47.

13. Barrey C, Jund J, Noseda O, Roussouly P. Sagittal balance of the pelvis-spine complex and lumbar degenerative diseases: a comparative study about 85 cases. Eur Spine J 2007;16:1459-67.

14. Lafage V, Schwab F, Vira S, Patel A, Ungar B, Farcy JP. Spino-pelvic parameters after surgery can be predicted: a preliminary formula and validation of standing alignment. Spine (Phila Pa 1976) 2011;36:1037-45.

15. Tanguay F, Mac-Thiong JM, de Guise JA, Labelle H. Relation between the sagittal pelvic and lumbar spine geometries following surgical correction of adolescent idiopathic scoliosis. Eur Spine J 2007;16:531-6.

16. Rothenfluh DA, Mueller DA, Rothenfluh E, Min K. Pelvic incidence-lumbar lordosis mismatch predisposes to adjacent segment disease after lumbar spinal fusion. Eur Spine J 2015;24:1251-8.

17. Bernhardt M, Bridwell KH. Segmental analysis of the sagittal plane alignment of the normal thoracic and lumbar spines and thoracolumbar junction. Spine (Phila Pa 1976) 1989;14:717-21.

18. Jackson RP, McManus AC. Radiographic analysis of sagittal plane alignment and balance in standing volunteers and patients with low back pain matched for age, sex, and size: a prospective controlled clinical study. Spine (Phila Pa 1976) 1994;19:1611-8.

19. Peterson M, Jackson R, McManus A. Standing sagittal spinal balance, alignments and lumbopelvic relationships: part I: a study of adult volunteers. Proceedings of the Annual Meeting of the Scoliosis Research Society; 1995; Asheville, USA. Milwaukee (WI): Scoliosis Research Society; 1995.

20. Kim WJ, Kang JW, Yeom JS, et al. A comparative analysis of sagittal spinal balance in 100 asymptomatic young and older aged volunteers. J Korean Soc 
Spine Surg 2003;10:327-34.

21. Aota Y, Kumano K, Hirabayashi S. Postfusion instability at the adjacent segments after rigid pedicle screw fixation for degenerative lumbar spinal disorders. J Spinal Disord 1995;8:464-73.

22. Kim HT, Kang DW, Yoo CH, Jeoung JH, Chang SA. Late changes at the adjacent segments to lumbar fusions. J Korean Soc Spine Surg 1996;3:1-10.

23. Brodsky AE, Hendricks RL, Khalil MA, Darden BV, Brotzman TT. Segmental ("floating") lumbar spine fusions. Spine (Phila Pa 1976) 1989;14:447-50.

24. Rohlmann A, Neller S, Bergmann G, Graichen F, Claes L, Wilke HJ. Effect of an internal fixator and a bone graft on intersegmental spinal motion and intradiscal pressure in the adjacent regions. Eur Spine J 2001;10:301-8.

25. Schwab F, Ungar B, Blondel B, et al. Scoliosis Research Society-Schwab adult spinal deformity classification: a validation study. Spine (Phila Pa 1976) 2012;37:1077-82.

26. Roussouly P, Gollogly S, Berthonnaud E, Dimnet J. Classification of the normal variation in the sagittal alignment of the human lumbar spine and pelvis in the standing position. Spine (Phila $\mathrm{Pa} 1976$ ) 2005;30:346-53. 